\title{
Experimental Results on Additive 2-Bases
}

\author{
By M. L. Stein and P. R. Stein
}

1. Introduction. This paper describes the construction of binary additive bases for all even numbers in some finite interval $2 \leqq 2 n \leqq N$. The construction makes use of a simple algorithm, first introduced by the authors in an earlier paper [1]. In the present paper the algorithm is applied to the sequence of primes, and several distinct "sparse" prime bases, constructed with its help, are described. As a byproduct of this work, the verification of the Goldbach conjecture has been extended up through all even numbers $2 n \leqq 10^{7}$. $\dagger$ The algorithm has also been applied to several random sequences of odd integers chosen so that their distribution is approximately that of the primes. Although the algorithm cannot, at present, be treated theoretically, even with regard to its asymptotic behavior, one may make plausible conjectures about it on the basis of various distinctive gross features; we hope to discuss this in a separate paper.

2. Definition of the Algorithm. Given a sequence of odd integers $\left\{a_{i}\right\}$, we wish to select a subsequence $\left\{b_{i}\right\}$ of these-generally with as few elements as will serve-so that every even number $2 n$ within certain limits, $N_{0} \leqq 2 n \leqq N_{1}$, can be written in the form:

$$
2 n=b_{i}+b_{j} .
$$

For example, the sequence $\left\{a_{i}\right\}$ may consist of the prime numbers greater than or equal to some initial prime $p_{0}=a_{0}$. In this case we can take the sequence $\left\{a_{i}\right\}$ to be as large as we like, introducing new members as they are needed; the number will depend on the upper limit $N_{1}$. We may equally well choose different sequences of odd numbers for our $\left\{a_{i}\right\}$-for example the sieve numbers known as "lucky numbers" [2], or, in fact, any set of odd numbers which can be generated according to a well-defined prescription.

Since our object is to produce a binary basis that will be in some sense "sparse," the following procedure immediately suggests itself. Let $b_{0}=a_{0}$. The first even number that can be expressed in the form (2.1) is $2 n_{0}=2 b_{0}$. For our second element we take $b_{1}=a_{1}$. To continue, form the even numbers $2 b_{0}, b_{0}+b_{1}, 2 b_{1}$. Let $2 n^{*}$ be the smallest even number $>2 b_{0}$ which does not belong to this set. We then look for the largest $a \in\left\{a_{i}\right\}$ such that either $b_{0}+a=2 n^{*}$ or $b_{1}+a=2 n^{*}$. If no such element exists, we move on to $2 n^{*}+2$, etc. More generally, given the partial basis $\left\{b_{0}, b_{1}, b_{2}, \cdots, b_{k}\right\}$, we form all the sums:

$$
S_{i j}=b_{i}+b_{j}
$$

$$
i \leqq j \leqq k \text {. }
$$

Next we find the smallest even number $2 n^{*}>N_{0}$ (where $N_{0}$ is the lower limit of the range) which does not belong to the set $\left\{S_{i j}\right\}$. We then set

$$
b_{k+1}=\underset{(a)}{\operatorname{Iax}_{a}}\left[a+b_{m}=2 n^{*}\right], \quad a \in\left\{a_{i}\right\}, 0 \leqq m \leqq k .
$$

Received December 21, 1964. This work was done under the auspices of the Atomic Energy Commission.

$†$ See Note added, page 433 of this issue. 
If this process is to yield a basis for the even numbers, then it must always be possible to find the "next" value $b_{k+1}$. If, for some even number $2 n^{*}$, it proves impossible to satisfy the equation $a+b_{i}=2 n^{*}(i \leqq k)$, we say that the algorithm "fails." In this case we replace $2 n^{*}$ by $2 n^{*}+2$ in equation (2.3) and proceed. If the equation cannot be satisfied for the even number $2 n^{*}+2$, we replace the latter by $2 n^{*}+4$, etc. Eventually a new basis element $b_{k+1}$ will be generated and the algorithm can be iterated.

It is clear that while the upper limit $N_{1}$ can be chosen in advance, the lower limit is a function of the set $\left\{a_{i}\right\}$; in fact, it is just the smallest even number such that the sequence $\left\{b_{i}\right\}$ forms a true basis for all the even numbers in the range $N_{0} \leqq 2 n \leqq N_{1}$. For "reasonable" sequences $\left\{a_{i}\right\}$ we expect that $N_{0}$ will be "close" to $2 a_{0}$; what this means in practice will become clear from a study of the numerical examples (see Tables I and III). If $\left\{a_{i}\right\}$ is the set of all primes (beginning with $a_{0}=3$ ) we would conjecture that the sequence $\left\{b_{i}\right\}$ generated by our algorithm is a binary additive basis for all even numbers $2 n \geqq 6$. At present, nothing further can be said about this "sharpened" form of Goldbach's conjecture; all our calculations show is that, with $\left\{a_{i}\right\}$ taken to be the set of primes less than $10^{7}$, the $\left\{b_{i}\right\}$ generated by our algorithm is, in fact, a basis for all the even numbers $6 \leqq 2 n \leqq 10^{7}$.

Suppose that, for some $N_{1}$ and a given set $\left\{a_{i}\right\}$, we have generated a basis $\left\{b_{i}\right\}$ for all the evens $N_{0} \leqq 2 n \leqq N_{1}$. Let us now fix $N_{0}$ and extend the upper limit $N_{1}$. (Note that we are in effect redefining $N_{0}$.) We cannot say that the algorithm will not fail somewhere between $N_{1}$ and the new upper limit $N_{2}>N_{1}$. If it does, however, our prescription still allows us to extend the sequence $\left\{b_{i}\right\}$, which would then no longer constitute a true basis. If the set $\left\{a_{i}\right\}$ is infinite we may let the upper limit approach infinity. The sequence $\left\{b_{i}\right\}$ is still well defined, and it would make sense to ask for an asymptotic formula for its density. A satisfactory treatment of this problem seems very desirable.

For the cases studied in this paper, every sequence $\left\{b_{i}\right\}$ has a density much less than that of the original sequence $\left\{a_{i}\right\}$. This would seem to justify using the term "sparse" to characterize the $\left\{b_{i}\right\}$. Accordingly, in the sequel we shall refer to the $\left\{b_{i}\right\}$ as "S-sequences" (or "S-bases") and to our algorithm as the "S-algorithm."

3. Results for the Prime Case. It is apparent from the above discussion that the S-sequence is uniquely determined by the set $\left\{a_{i}\right\}$. In particular, the elements of $\left\{b_{i}\right\}$ depend critically on the value of $a_{0}$. Let $\left\{a_{i}\right\}$ be the sequence of (odd) primes, starting with a particular prime $a_{0}=p_{0}$. Then different sequences $\left\{b_{i}\right\}$ will be produced by different choices of $p_{0}$. In the sequel we shall distinguish these different sequences by adding a subscript; thus the sequence corresponding to a particular choice of $p_{0}$ will be denoted by $\left\{b_{i}\right\}_{p_{0}}$. As an example, take the two sequences corresponding to $p_{0}=3, p_{0}=11$, respectively. The first 22 terms of $\left\{b_{i}\right\}_{3}$ are: $3,5,7,13$, $19,23,31,37,43,47,53,61,79,83,109,113,101,131,139,157,167,199$. For $\left\{b_{i}\right\}_{11}$ the first 22 terms are: $11,13,17,19,29,31,41,43,53,37,59,79,73,113,109$, $103,107,151,163,167,179,191$. Note that the elements $b_{i}$ are not produced in strictly ascending order. We have called this phenomenon "backtracking." Thus, in the case $p_{0}=3$, the first 16 terms constitute a 2-basis for the even numbers $6 \leqq 2 n \leqq 122$. To express $2 n=124$ in the required form we must introduce the 
prime $b_{16}=101: 124=101+23$. While backtracking appears to persist even when one goes to higher values, it is not very prevalent. For example in the S-sequences for $p_{0}=1,3$, and 5 , the number of elements less than 5 million which are generated "out of order" is, respectively, $5.55 \%, 6.23 \%$ and $5.22 \%$ of the total.

Let $B\left(p_{0} ; x\right)$ be the number of primes $\leqq x$ in the S-sequence $\left\{b_{i}\right\}_{p_{0}}$. It is of interest to compare the values of $B\left(p_{0} ; x\right)$ for a set of equally spaced $x$-values and different $p_{0}$. In Table I such a comparison is exhibited at intervals $\Delta x=200,000, x \leqq 5 \times 10^{6}$. In each case, the $\left\{b_{i}\right\}_{p_{0}}$ was found to constitute a true binary additive basis for all $N_{0} \leqq 2 n \leqq x$; in other words, with the exception of a few early values $\left(2 n<N_{0}\right)$, no failure was observed in any case.

Leaving aside for the moment the anomalous case $p_{0}=7$ (see Section 5 ), it is quite remarkable how small the variation of $B\left(p_{0} ; x\right)$ with $p_{0}$ is for fixed $x$; in the second half of the table $\left(x \geqq 24 \times 10^{5}\right)$ the absolute spread is less than $1.5 \%$ of the lowest value for each $x$ listed. This is perhaps all the more remarkable in view of the fact that the various S-bases $\left\{b_{i}\right\}_{p_{0}}$ are very nearly pairwise disjoint, the number of primes common to two different sequences being typically between $3 \%$ and $4 \%$ of the total number in the shorter sequence. Let $C(u, v ; x)$ be the number of primes common to the two S-sequences $\left\{b_{i}\right\}_{u}$ and $\left\{b_{i}\right\}_{v}$ for the range $2 n \leqq x$. Tables II-a and II-b give a partial tabulation of $C(u, v ; x)$ for the range $2 n \leqq 5 \times$ $10^{6}$. In two cases- $p_{0}=1$ and $p_{0}=3$-the S-sequence has been calculated up to $2 n=10^{7}$. In these cases, we find $B\left(1 ; 10^{7}\right)=10474, B\left(3 ; 10^{7}\right)=10576$. For this range, the number of primes common to these two sequences is $C\left(1,3 ; 10^{7}\right)=288$. In passing we remark that this calculation verifies the Goldbach conjecture for all even numbers $2 n \leqq 10^{7}$; to achieve this basis, less than $1.6 \%$ of the available primes are required.

4. Random Odds. As remarked in Section 2, the S-algorithm is not restricted to the sequence of primes. For example, in [1] we reported the construction of an S-basis for the evens $2 n \leqq 350,000$ which was composed of lucky numbers. This basis was found to consist of 1672 luckies out of a total of 27420 luckies in the range. More recently, we have applied the algorithm to sets of odd numbers with approximately "prime-like" distribution. These sets were generated as follows. Let $p_{i}$ be the $i$ th prime. We chose at random 360 odd numbers equally distributed in the interval 3 to $p_{360}$, then 360 more in the interval $p_{361}$ to $p_{720}$, and so forth up to $p_{78498}$, the last prime less than $10^{6}$ (the number of odds in the final interval was suitably adjusted). Five such random sets were generated; we shall denote them by the symbols $\mathrm{RO}(1), \mathrm{RO}(2), \cdots, \mathrm{RO}(5)$; for the conclusions we will draw here it is not necessary to specify them more fully. To each of these sets we then applied the S-algorithm. To facilitate comparison with the "standard" S-sequence $\left\{b_{i}\right\}_{3}$, we forced the first odd in each case to be $a_{0}=3$. Let us denote by $R B_{j}(x)$ the number of elements $\leqq x$ in the S-sequence generated from the set $\mathrm{RO}(j)$. In Table III we have tabulated $R B_{j}(x)$ for our five sets at ten equally spaced values of $x \leqq 10^{6}$. The last column gives $B(3 ; x)$ for comparison.

The agreement for given $x$ is remarkable, especially in view of the fact that there is no connection between the random sets $\mathrm{RO}(j)$ beyond their common primelike distribution. As one might expect, the S-sequences corresponding to any two 
TABLE I

\begin{tabular}{|c|c|c|c|c|c|c|}
\hline $10^{-5} x$ & $\begin{array}{c}B(1 ; x) \\
\left(N_{0}=2\right)\end{array}$ & $\begin{array}{c}B(3 ; x) \\
\left(N_{0}=6\right)\end{array}$ & $\begin{array}{c}B(5 ; x) \\
\left(N_{0}=10\right)\end{array}$ & $\begin{array}{c}B(7 ; x) \\
\left(N_{0}=18\right)\end{array}$ & $\begin{array}{c}B(11 ; x) \\
\left(N_{0}=22\right)\end{array}$ & $\begin{array}{c}B(13 ; x) \\
\left(N_{0}=30\right)\end{array}$ \\
\hline 2 & 1235 & 1245 & 1263 & 1287 & 1236 & 1233 \\
\hline 4 & 1820 & 1837 & 1844 & 1970 & 1822 & 1813 \\
\hline 6 & 2265 & 2288 & 2300 & 2566 & 2268 & 2264 \\
\hline 8 & 2658 & 2681 & 2688 & 3089 & 2663 & 2654 \\
\hline 10 & 3000 & 3029 & 3027 & 3588 & 3005 & 3008 \\
\hline 12 & 3307 & 3337 & 3360 & 4074 & 3300 & 3315 \\
\hline 14 & 3598 & 3623 & 3640 & 4508 & 3599 & 3613 \\
\hline 16 & 3868 & 3909 & 3919 & 4955 & 3881 & 3882 \\
\hline 18 & 4123 & 4178 & 4178 & 5344 & 4130 & 4131 \\
\hline 20 & 4377 & 4421 & 4416 & 5747 & 4382 & 4390 \\
\hline 22 & 4612 & 4661 & 4656 & 6133 & 4615 & 4610 \\
\hline 24 & 4826 & 4883 & 4882 & 6514 & 4837 & 4832 \\
\hline 26 & 5057 & 5108 & 5087 & 6875 & 5048 & 5050 \\
\hline 28 & 5268 & 5318 & 5298 & 7216 & 5251 & 5250 \\
\hline 30 & 5472 & 5517 & 5505 & 7568 & 5454 & 5458 \\
\hline 32 & 5656 & 5710 & 5699 & 7892 & 5647 & 5643 \\
\hline 34 & 5857 & 5880 & 5891 & 8210 & 5832 & 5828 \\
\hline 36 & 6045 & 6078 & 6071 & 8523 & 6021 & 6023 \\
\hline 38 & 6218 & 6271 & 6260 & 8820 & 6204 & 6205 \\
\hline 40 & 6405 & 6452 & 6426 & 9132 & 6383 & 6387 \\
\hline 42 & 6568 & 6609 & 6598 & 9450 & 6548 & 6550 \\
\hline 44 & 6733 & 6787 & 6749 & 9741 & 6719 & 6707 \\
\hline 46 & 6896 & 6948 & 6920 & 10020 & 6889 & 6877 \\
\hline 48 & 7057 & 7110 & 7086 & 10299 & 7045 & 7029 \\
\hline 50 & 7211 & 7274 & 7256 & 10579 & 7220 & 7186 \\
\hline $10^{-5} x$ & $\begin{array}{c}B(71 ; x) \\
\left(N_{0}=166\right)\end{array}$ & $\begin{array}{c}B(73 ; x) \\
\left(N_{0}=166\right)\end{array}$ & $\begin{array}{c}B(79 ; x) \\
\left(N_{0}=176\right)\end{array}$ & $\begin{array}{c}B(83 ; x) \\
\left(N_{0}=190\right)\end{array}$ & $\begin{array}{c}B(89 ; x) \\
\left(N_{0}=190\right)\end{array}$ & $\begin{array}{c}B(97 ; x) \\
\left(N_{0}=198\right)\end{array}$ \\
\hline 2 & 1231 & 1239 & 1247 & 1239 & 1246 & 1239 \\
\hline 4 & 1803 & 1814 & 1809 & 1821 & 1811 & 1815 \\
\hline 6 & 2259 & 2266 & 2267 & 2277 & 2263 & 2270 \\
\hline 8 & 2644 & 2652 & 2657 & 2661 & 2644 & 2660 \\
\hline 10 & 2984 & 2996 & 2991 & 3002 & 2994 & 2989 \\
\hline 12 & 3294 & 3308 & 3317 & 3319 & 3308 & 3302 \\
\hline 14 & 3580 & 3593 & 3609 & 3595 & 3606 & 3594 \\
\hline 16 & 3864 & 3861 & 3885 & 3884 & 3875 & 3867 \\
\hline 18 & 4125 & 4120 & 4139 & 4127 & 4128 & 4117 \\
\hline 20 & 4359 & 4368 & 4371 & 4374 & 4368 & 4366 \\
\hline 22 & 4603 & 4609 & 4603 & 4610 & 4614 & 4589 \\
\hline 24 & 4831 & 4824 & 4832 & 4835 & 4829 & 4813 \\
\hline 26 & 5042 & 5045 & 5046 & 5046 & 5035 & 5024 \\
\hline 28 & 5254 & 5252 & 5255 & 5247 & 5246 & 5236 \\
\hline 30 & 5455 & 5460 & 5449 & 5459 & 5448 & 5437 \\
\hline 32 & 5636 & 5642 & 5638 & 5658 & 5640 & 5627 \\
\hline 34 & 5835 & 5831 & 5817 & 5840 & 5833 & 5814 \\
\hline 36 & 6021 & 6017 & 6012 & 6015 & 6020 & 6008 \\
\hline 38 & 6201 & 6190 & 6192 & 6215 & 6188 & 6180 \\
\hline 40 & 6376 & 6378 & 6374 & 6377 & 6369 & 6358 \\
\hline 42 & 6539 & 6537 & 6544 & 6553 & 6534 & 6528 \\
\hline 44 & 6720 & 6707 & 6704 & 6721 & 6703 & 6696 \\
\hline 46 & 6876 & 6866 & 6872 & 6876 & 6869 & 6860 \\
\hline 48 & 7031 & 7021 & 7033 & 7028 & .7027 & 7028 \\
\hline 50 & 7181 & 7177 & 7185 & 7182 & 7186 & 7178 \\
\hline
\end{tabular}


TABLE II-a

\begin{tabular}{r|c|c|c|c}
\hline \multirow{2}{*}{$u$} & \multicolumn{4}{|c}{$v$} \\
\cline { 2 - 5 } & 3 & 5 & 11 & 13 \\
\hline 1 & 250 & 253 & 282 & 269 \\
3 & & 251 & 263 & 258 \\
5 & & & 237 & 275 \\
11 & & & & 274 \\
& & & & \\
\hline
\end{tabular}

TABLE II-b

\begin{tabular}{c|c|c|c|c|c}
\hline & \multicolumn{5}{|c}{$v$} \\
\cline { 3 - 6 } & 73 & 79 & 83 & 89 & 97 \\
\hline 71 & 264 & 253 & 280 & 259 & 290 \\
73 & & 284 & 244 & 269 & 286 \\
79 & & & 272 & 269 & 239 \\
83 & & & & 301 & 300 \\
89 & & & & & 281 \\
\hline
\end{tabular}

TABLE III

\begin{tabular}{c|c|c|c|c|c|c}
\hline $10^{-5} x$ & $\begin{array}{c}R B_{1}(x) \\
\left(N_{0}=38\right)\end{array}$ & $\begin{array}{c}R B_{2}(x) \\
\left(N_{0}=16\right)\end{array}$ & $\begin{array}{c}R B_{3}(x) \\
\left(N_{0}=136\right)\end{array}$ & $\begin{array}{c}R B_{4}(x) \\
\left(N_{0}=52\right)\end{array}$ & $\begin{array}{c}R B_{5}(x) \\
\left(N_{0}=158\right)\end{array}$ & $B(3 ; x)$ \\
\cline { 2 - 5 } 1 & 754 & 757 & 760 & 763 & 760 & 843 \\
2 & 1115 & 1113 & 1113 & 1120 & 1116 & 1245 \\
3 & 1392 & 1384 & 1382 & 1389 & 1383 & 1565 \\
4 & 1619 & 1626 & 1624 & 1623 & 1618 & 1837 \\
5 & 1826 & 1826 & 1835 & 1830 & 1826 & 2075 \\
6 & 2025 & 2023 & 2018 & 2023 & 2016 & 2288 \\
7 & 2200 & 2205 & 2193 & 2197 & 2186 & 2494 \\
8 & 2370 & 2364 & 2361 & 2379 & 2361 & 2681 \\
9 & 2530 & 2516 & 2523 & 2526 & 2516 & 2862 \\
10 & 2676 & 2663 & 2661 & 2673 & 2666 & 3029 \\
\hline
\end{tabular}

random sets $\mathrm{RO}(i), \mathrm{RO}(j)$ have very few common elements. For the pair $\mathrm{RO}(1)$, $\mathrm{RO}(2)$, the S-sequences have 117 elements in common; for the other nine pairs the number of common elements varies between 23 and 39 .

It is noteworthy that these prime-like random sets give rise to S-sequences markedly sparser than those produced by the primes themselves. The $\mathrm{RO}(i)$ are, however, prime-like only with respect to their overall density. For example, the distribution of gaps between successive elements is quite different from that which obtains for the prime sequence. In Table IV we compare the prime gap distribution for gaps of size $g \leqq 56$ (between successive primes) with the corresponding distribution for four of our random sets (range: $a_{i} \leqq 10^{6}$ ); in this table, $N(g)$ denotes the number of gaps of size $g$ between successive elements. The complete absence of "modulo 6 peaks" and the consequent monotonic decrease of $N(g)$ with increasing $g$ are just what one would expect. In view of the results presented in Table III, we may say that the primes, far from being a "privileged" sequence with regard to their efficiency as a binary additive basis for the evens, are somehow handicapped because of the distribution imposed on them by their defining sieve.

The greater "efficiency" of the S-sequences generated from our random odd sets is also mirrored in the corresponding "Goldbach frequency distribution." This distribution may be defined for prime S-sequences as follows. Let $\nu\left(p_{0} ; 2 n\right)$ be the number of solutions of the equation

$$
2 n=b_{i}+b_{j}, \quad i \leqq j, b_{i}, b_{j} \in\left\{b_{i}\right\}_{p_{0}} .
$$


TABLE IV

Number of Gaps $N(g)$ of Size $g$ Between Consecutive Elements range: $a_{i} \leqq 10^{6}$

\begin{tabular}{|c|c|c|c|c|c|}
\hline$g$ & $\begin{array}{c}N(g) \\
\text { (Primes) }\end{array}$ & $\begin{array}{c}N(g) \\
{[\mathrm{RO}(2)]}\end{array}$ & $\begin{array}{c}N(\xi) \\
{[\mathrm{RO}(3)]}\end{array}$ & $\begin{array}{c}N(g) \\
{[\mathrm{RO}(4)]}\end{array}$ & $\begin{array}{c}N(g) \\
{[\operatorname{RO}(5)]}\end{array}$ \\
\hline 2 & 8169 & 12412 & 12469 & 12325 & 12532 \\
\hline 4 & 8143 & 10387 & 10429 & 10529 & 10411 \\
\hline 6 & 13549 & 8827 & 8808 & 8777 & 8744 \\
\hline 8 & 5569 & 7271 & 7298 & 7482 & 7299 \\
\hline 10 & 7079 & 6245 & 6308 & 6289 & 6258 \\
\hline 12 & 8005 & 5300 & ' 5156 & 5192 & 5274 \\
\hline 14 & 4233 & 4355 & 4376 & 4230 & 4374 \\
\hline 16 & 2881 & 3630 & 3666 & 3670 & 3593 \\
\hline 18 & 4909 & 3090 & 3163 & 3078 & 3111 \\
\hline 20 & 2401 & 2583 & 2605 & 2629 & 2627 \\
\hline 22 & 2172 & 2316 & 2250 & 2246 & 2243 \\
\hline 24 & 2682 & 1909 & 1819 & 1895 & 1901 \\
\hline 26 & 1175 & 1599 & 1557 & 1581 & 1543 \\
\hline 28 & 1234 & 1289 & 1333 & 1354 & 1301 \\
\hline 30 & 1914 & 1181 & 1123 & 1142 & 1142 \\
\hline 32 & 550 & 938 & 941 & 943 & 935 \\
\hline 34 & 557 & 807 & 761 & 775 & 824 \\
\hline 36 & 767 & 702 & 721 & 669 & 701 \\
\hline 38 & 330 & 585 & 579 & 570 & 556 \\
\hline 40 & 424 & 493 & 438 & 452 & 494 \\
\hline 42 & 476 & 438 & 416 & 426 & 415 \\
\hline 44 & 202 & 362 & 366 & 333 & 339 \\
\hline 46 & 155 & 273 & 306 & 307 & 284 \\
\hline 48 & 196 & 237 & 231 & 255 & 250 \\
\hline 50 & 106 & 205 & 229 & 216 & 222 \\
\hline 52 & 77 & 148 & 160 & 166 & 162 \\
\hline 54 & 140 & 134 & 148 & 159 & 139 \\
\hline 56 & 53 & 122 & 142 & 123 & 126 \\
\hline
\end{tabular}

By the "Goldbach frequency distribution at the point $(k, x)$ " we mean the number of solutions $\sigma_{k}\left(p_{0} ; x\right)$ of the equation

$$
\nu\left(p_{0} ; 2 n\right)=k, \quad 2 n \leqq x .
$$

A corresponding definition holds for the random odd S-sequences, where we replace the label $p_{0}$ by an appropriate symbol characterizing the underlying random odd set. In general, the random odd S-sequences have frequency distributions which are much more peaked (as a function of $k$ for fixed $x$ ) than those belonging to the prime S-sequences. For example, if we form the sum $\sum_{k=1}^{10} \sigma_{k}\left(p_{0} ; 10^{6}\right)$ for any of the prime S-sequences (excluding $p_{0}=7$ ), we find that we have included approximately $83 \%$ of the total number of decompositions; the corresponding number for the random odd $\mathrm{S}$-sequences is about $98 \%$.

5. The Anomalous Case. It is evident from Table I that $\left\{b_{i}\right\}_{7}$ is much denser than any of the other prime $\mathbf{S}$-sequences studied. The result is so anomalous that one is 
TABLE $\mathrm{V}$

\begin{tabular}{c|c}
\hline$p_{0}$ & Number of evens $2 n^{*}$ of the form $6 m$ \\
\hline 1 & 33 \\
3 & 45 \\
5 & 30 \\
7 & 18 \\
11 & 33 \\
71 & 42 \\
73 & 38 \\
79 & 27 \\
83 & 30 \\
89 & 30 \\
97 & 31 \\
\hline
\end{tabular}

led to suspect a calculational error; numerous independent checks, however, have failed to turn up anything of the sort. So far as we can tell, the observed behavior is simply a numerical accident. There is, however, one property of the S-algorithm which may shed some light on the nature of this accident. It happens that, for all $p_{0}$ tried so far, the even numbers $2 n^{*}$ which determine the successive $b_{i}$ (see equation (2.3)) are very rarely divisible by 6 . This is shown in Table $\mathrm{V}$ for the range $2 n \leqq 5$ $\times 10^{6}$. This behavior itself remains to be explained, but given this observed property it is not unreasonable that a sufficiently large asymmetry in the distribution of the $b_{i}$ modulo 3 will increase in magnitude rather than be damped out. Such behavior would clearly lead to a much denser sequence $\left\{b_{i}\right\}$ and perhaps even to eventual failure of the algorithm. As it happens, all our prime S-sequences except that for $p_{0}=7$ are evenly distributed $(\bmod 3)$. The anomalous sequence, however, shows a ratio of 1.93 between primes $\equiv 2(\bmod 3)$ and primes $\equiv 1(\bmod 3)$. This is for the interval $2 n \leqq 5 \times 10^{6}$. The sequence $\left\{b_{i}\right\}_{7}$ was actually computed up to $2 n=7 \times$ $10^{6}$; here $B\left(7 ; 7 \times 10^{6}\right)=13108$ and the above-mentioned ratio has risen to 2.04 . We have watched the development of this asymmetry in some detail without, however, learning anything whatsoever about the underlying reason for the anomalous behavior.

Other distinctive properties of $\left\{b_{i}\right\}_{7}$ are consistent with the observed behavior of $B(7 ; x)$. The Goldbach frequency distribution $\sigma_{k}(7 ; x)$ is much broader (as a function of $k$ ) than it is for any of the other cases studied. In addition, the "backtracking" phenomenon mentioned in Section 2 is much more pronounced for this case; in the range $2 n \leqq 5 \times 10^{6}$, some $9.95 \%$ of the minimals were generated "out of order."

Note added in proof. Since this article was written, we have extended our verification of the Goldbach conjecture up to one hundred million, using a simple sieve technique quite independent of the S-sequence method reported here. As a result of this work we may state the following-not very surprising-empirical theorem.

Let $p=P(2 n)$ be the smallest odd prime $\geqq 3$ such that $2 n-p$ is a prime. Then, for $63276 \leqq 2 n \leqq 10^{8}, P(2 n)<\sqrt{2 n}$. For this range, the maximum value of $P(2 n)$ turns out to be 1093: $60119912=1093+60118819$.

At the suggestion of Dr. D. Shanks, we also carried through the verification, 
over the same range, of the "modified" Goldbach conjecture, namely that every even number $4 n+2$ is the sum of two primes of the form $4 k+1$ (here 1 is counted as a prime). The above theorem holds, mutatis mutandis, for this case also, i.e. for $1457284 \leqq 2 n(=4 m+2)<10^{8}, P(2 n)<\sqrt{2 n}$. In this case, the maximum value of $P(2 n)$ over the range is $2953: 76550462=2953+76547509$.

Los Alamos Scientific Laboratory

Los Alamos, New Mexico

1. M. L. Stein \& P. R. STEIN, "New experimental results on the Goldbach conjecture," Math. Mag., v. 38, 1965, pp. 72-80.

2. V. Gardiner, R. Lazarus, N. Metropolis \& S. UlaM, "On certain sequences of integers defined by sieves," Math. Mag., v. 29, 1956, pp. 117-122. MR 17, 711. 